\title{
Strong convergence theorems for a finite family of total asymptotically strict pseudocontractive semigroups in Banach spaces
}

Li Yang $^{1 *}$, Shih-sen Chang ${ }^{2}$ and Fu Hai Zhao ${ }^{1}$

"Correspondence:

yanglizxs@yahoo.com.cn ${ }^{1}$ School of Science, South West

University of Science and Technology, Mianyang, Sichuan 621002, China

Full list of author information is available at the end of the article

\begin{abstract}
The purpose of this paper is to introduce the concepts of total asymptotically strictly pseudocontractive semigroup, asymptotically strictly pseudocontractive semigroup etc., and to prove some strong convergence theorems of the explicit iteration process for these kinds of semigroups in arbitrary Banach spaces. The results presented in the paper extend and improve some recent results announced in the current literature.

MSC: $47 \mathrm{H} 20 ; 47 \mathrm{H} 10$

Keywords: total asymptotically strict pseudocontractive semigroup; asymptotically strict pseudocontractive semigroup; asymptotically demicontractive semigroup; fixed point; normalized duality mapping
\end{abstract}

\section{Introduction and preliminaries}

Let $E$ be a real Banach space, $E^{*}$ be the dual space of $E$. In the sequel we assume that $C$ is a nonempty closed convex subset of $E, \Re^{+}$is the set of nonnegative real numbers and $J: E \rightarrow 2^{E^{*}}$ is the normalized duality mapping defined by

$$
J(x)=\left\{f \in E^{*}:\langle x, f\rangle=\|x\| \cdot\|f\|,\|x\|=\|f\|\right\}, \quad x \in E .
$$

Let $T: C \rightarrow C$ be a mapping, we denote by $F(T)$ the set of fixed points of $T$. We also use ' $\rightarrow$ ' to stand for strong convergence and ' $\rightarrow$ ' for weak convergence.

We first recall some definitions.

A one-parameter family $\mathfrak{s}:=\{T(t): t \geq 0\}$ of self-mappings of $C$ is said to be a nonexpansive semigroup if the following conditions are satisfied:

(i) $T\left(t_{1}+t_{2}\right) x=T\left(t_{1}\right) T\left(t_{2}\right) x$, for any $t_{1}, t_{2} \in \Re^{+}$and $x \in C$;

(ii) $T(0) x=x$ for each $x \in C$;

(iii) for each $x \in C, t \mapsto T(t) x$ is continuous;

(iv) for any $t \geq 0, T(t)$ is a nonexpansive mapping on $C$, that is, for any $x, y \in C$,

$$
\|T(t) x-T(t) y\| \leq\|x-y\| .
$$

If the family $\mathfrak{I}:=\{T(t): t \geq 0\}$ satisfies conditions (i)-(iii), then it is: 
(a) Pseudocontractive semigroup if for any $x, y \in C$, there exists $j(x-y) \in J(x-y)$ such that

$$
\langle T(t) x-T(t) y, j(x-y)\rangle \leq\|x-y\|^{2} .
$$

(b) Uniformly Lipschitzian semigroup if there exists a bounded measurable function $L$ : $[0, \infty) \rightarrow(0, \infty)$ such that, for any $x, y \in C$ and $t \geq 0$,

$$
\left\|T^{n}(t) x-T^{n}(t) y\right\| \leq L(t)\|x-y\|, \quad \forall n \geq 1 .
$$

In this case, we also say that $\Im$ is a uniformly $L(t)$-Lipschitzian semigroup.

(c) Strictly pseudocontractive semigroup if there exists a bounded function $\lambda:[0, \infty) \rightarrow$ $(0, \infty)$ and for any given $x, y \in C$, there exists $j(x-y) \in J(x-y)$ such that

$$
\langle T(t) x-T(t) y, j(x-y)) \mid \leq\|x-y\|^{2}-\lambda(t)\|(I-T(t)) x-(I-T(t)) y\|^{2}
$$

for any $t \geq 0$.

It is easy to see that such a semigroup is $((1+\lambda(t)) / \lambda(t))$-Lipschitzian and pseudocontractive semigroup.

(d) Demicontractive semigroup if $\bigcap_{t \geq 0} F(T(t)) \neq \emptyset$, and there exists a bounded function $\lambda:[0, \infty) \rightarrow(0, \infty)$ for any $t \geq 0, x \in C$ and $y \in \bigcap_{t \geq 0} F(T(t))$, there exists $j(x-y) \in J(x-y)$ such that

$$
\langle T(t) x-y, j(x-y)| \leq\|x-y\|^{2}-\lambda(t)\|(I-T(t)) x\|^{2} .
$$

In this paper, we introduce the following semigroups.

Definition 1.1 A one-parameter family $\Im:=\{T(t): t \geq 0\}$ of self-mappings of $C$ satisfies conditions (i)-(iii), then it is:

(e) Total asymptotically strictly pseudocontractive semigroup if there exist a bounded function $\lambda:[0, \infty) \rightarrow(0, \infty)$ and sequences $\left\{\mu_{n}\right\} \subset[0, \infty)$ and $\left\{\xi_{n}\right\} \subset[0, \infty)$ with $\mu_{n} \rightarrow 0$ and $\xi_{n} \rightarrow 0$ as $n \rightarrow \infty$. For any given $x, y \in C$, there exists $j(x-y) \in J(x-y)$ such that

$$
\begin{aligned}
\left\langle T^{n}(t) x-T^{n}(t) y, j(x-y)\right\rangle \leq & \|x-y\|^{2}-\lambda(t)\left\|\left(I-T^{n}(t)\right) x-\left(I-T^{n}(t)\right) y\right\|^{2} \\
& +\mu_{n} \phi(\|x-y\|)+\xi_{n}, \quad \forall n \geq 1, \forall t \geq 0,
\end{aligned}
$$

where $\phi:[0, \infty) \rightarrow[0, \infty)$ is a continuous and strictly increasing function with $\phi(0)=0$.

In this case, we also say that $\Im$ is a $\left(\lambda(t),\left\{\mu_{n}\right\},\left\{\xi_{n}\right\}, \phi\right)$-total asymptotically strict pseudocontractive semigroup.

(f) Asymptotically strictly pseudocontractive semigroup if there exist a bounded function $\lambda:[0, \infty) \rightarrow(0, \infty)$ and a sequence $\left\{k_{n}\right\} \subset[1, \infty)$ with $k_{n} \rightarrow 1$ as $n \rightarrow \infty$; for any given $x, y \in C$, there exists $j(x-y) \in J(x-y)$ such that

$$
\begin{aligned}
& \left\langle T^{n}(t) x-T^{n}(t) y, j(x-y)\right\rangle \\
& \quad \leq k_{n}\|x-y\|^{2}-\lambda(t)\left\|\left(I-T^{n}(t)\right) x-\left(I-T^{n}(t)\right) y\right\|^{2}, \quad \forall n \geq 1,
\end{aligned}
$$

for any $t \geq 0$. 
In this case, we also say that $\Im$ is a $\left(\left\{k_{n}\right\}, \lambda(t)\right)$-asymptotically strict pseudocontractive semigroup.

(g) Asymptotically demicontractive semigroup if $\bigcap_{t \geq 0} F(T(t)) \neq \emptyset$ and there exist a bounded function $\lambda:[0, \infty) \rightarrow(0, \infty)$ and a sequence $\left\{k_{n}\right\} \subset[1, \infty)$ with $k_{n} \rightarrow 1$ as $n \rightarrow \infty$; for any $t \geq 0, x \in C$ and $y \in \bigcap_{t \geq 0} F(T(t))$, there exists $j(x-y) \in J(x-y)$ such that

$$
\left\langle T^{n}(t) x-y, j(x-y)\right\rangle \leq k_{n}\|x-y\|^{2}-\lambda(t)\left\|\left(I-T^{n}(t)\right) x\right\|^{2}, \quad \forall n \geq 1,
$$

for any $t \geq 0$.

In this case, we also say that $\Im$ is a $\left(\left\{k_{n}\right\}, \lambda(t)\right)$-asymptotically demicontractive semigroup.

Remark 1.2 If $\phi(\lambda)=\lambda^{2}$ and $\xi_{n}=0$, then a total asymptotically strict pseudocontractive semigroup is an asymptotically strict pseudocontractive semigroup. Every asymptotically strict pseudocontractive semigroup with $\bigcap_{t>0} F(T(t)) \neq \phi$ is an asymptotically demicontractive semigroup. If $k_{n}=1, \forall n \geq 1$, an asymptotically strict pseudocontractive semigroup is a strict pseudocontractive semigroup, an asymptotically demicontractive semigroup is a demicontractive semigroup.

It is easy to see that condition (1.7) is equivalent to the following condition: for any $t \geq 0$, $x \in C$ and $y \in F(T(t))$, there exists $j(x-y) \in J(x-y)$ such that

$$
\left\langle x-T^{n}(t) x, j(x-y)\right\rangle \geq \lambda(t)\left\|x-T^{n}(t) x\right\|^{2}-\mu_{n} \phi(\|x-y\|)-\xi_{n} .
$$

The convergence problem of implicit and explicit iterative sequences for nonexpansive semigroups to a common fixed point has been considered by some authors in various spaces; see, for example, [1-11].

In 1998, Shioji-Takahashi [1] introduced and studied a Halpern-type scheme for a common fixed point of a family of asymptotically nonexpansive semigroups in the framework of a real Hilbert space.

In 2003, Suzuki [2] proved that the implicit scheme defined by $u, x_{1} \in C$

$$
x_{n}=\alpha_{n} u+\left(1-\alpha_{n}\right) T\left(t_{n}\right)\left(x_{n}\right), \quad n \geq 1,
$$

converges strongly to a common fixed point of the family of nonexpansive semigroups in a real Hilbert space. Xu [3] extended the result of Suzuki to a more general real uniformly convex Banach space having a weakly sequentially continuous duality mapping.

In 2005, Aleyner and Reich [4] proved the strong convergence of an explicit Halperntype scheme defined by $u, x_{1} \in C$

$$
x_{n+1}=\alpha_{n} u+\left(1-\alpha_{n}\right) T\left(t_{n}\right) x_{n}, \quad n \geq 0,
$$

to a common fixed point of the family $\{T(t): t \geq 0\}$ of nonexpansive semigroups in a reflexive Banach space with a uniformly Gâteaux differentiable norm.

Recently, Chang et al. [11] introduced the following explicit iteration process:

$$
\begin{aligned}
& x_{1} \in C, \\
& x_{n+1}=\left(1-\alpha_{n}\right) x_{n}+\alpha_{n} T\left(t_{n}\right) x_{n}, \quad n \geq 1,
\end{aligned}
$$


for the Lipschitzian and demicontractive semigroup $\mathfrak{\Im}:=\{T(t): t \geq 0\}$ in general Banach spaces. Under appropriate assumptions imposed upon the parameter sequences $\left\{\alpha_{n}\right\}$ and $\left\{t_{n}\right\}$, they proved that the sequence $\left\{x_{n}\right\}$ defined by (1.13) converges strongly to some point in $F=: \bigcap_{t \geq 0} F(T(t))$.

Inspired and motivated by the above works of Shioji-Takahashi [1], Suzuki [2], Xu [3], Aleyner-Reich [4] and Chang [11], in this paper we aim to study the strong convergence to a common fixed point for a finite family of uniformly Lipschitzian and total asymptotically strict pseudocontractive semigroups $\Im_{i}:=\left\{T_{i}(t): t \geq 0\right\}, \forall i \in\{1,2, \ldots, N\}$ in general Banach spaces. The results presented in the paper extend and improve some recent results given in $[4,5,7,9]$.

The following lemmas will be needed in proving our main results.

Lemma 1.3 Let $\left\{a_{n}\right\},\left\{b_{n}\right\}$ and $\left\{\delta_{n}\right\}$ be sequences of nonnegative real numbers satisfying

$$
a_{n+1} \leq\left(1+\delta_{n}\right) a_{n}+b_{n}, \quad \forall n \geq n_{0},
$$

where $n_{0}$ is some nonnegative integer. If $\sum_{n=1}^{\infty} \delta_{n}<\infty$ and $\sum_{n=1}^{\infty} b_{n}<\infty$, then the limit $\lim _{n \rightarrow \infty} a_{n}$ exists.

Lemma 1.4 [12] Let $E$ be any real Banach space, let $E^{*}$ be the dual space of $E$ and let $J: E \rightarrow 2^{E^{*}}$ be the normalized duality mapping. Then, for any $x, y \in E$, we have

$$
\|x+y\|^{2} \leq\|x\|^{2}+2(y, j(x+y)), \quad \forall j(x+y) \in J(x+y) .
$$

\section{Main results}

Let $E$ be a real Banach space, and let $C$ be a nonempty, closed convex subset of $E$. For some fixed $i \in N$, let $\Im_{i}:=\left\{T_{i}(t): t \geq 0\right\}$ be a uniformly $L_{i}(t)$-Lipschitzian with a bounded measurable function $L_{i}:[0, \infty) \rightarrow(0, \infty)$ and $\left(\lambda_{i}(t),\left\{\mu_{i n}\right\},\left\{\xi_{i n}\right\}, \phi_{i}\right)$-total asymptotically strictly pseudocontractive semigroup with a bounded function $\lambda_{i}:[0, \infty) \rightarrow(0, \infty)$, sequences $\left\{\mu_{\text {in }}\right\},\left\{\xi_{\text {in }}\right\} \subset[0, \infty)$ with $\mu_{\text {in }} \rightarrow 0$ and $\xi_{\text {in }} \rightarrow 0$ as $n \rightarrow \infty$, such that

$$
L^{i}:=\sup _{t \geq 0} L_{i}(t)<\infty, \quad \lambda^{i}:=\inf _{t \geq 0} \lambda_{i}(t)>0, \quad F^{i}:=\bigcap_{t \geq 0} F\left(T_{i}(t)\right) \neq \emptyset .
$$

Then, for $x, y \in C, p \in F^{i}$ and $t \geq 0$,

$$
\begin{aligned}
\left\langle T_{i}^{n}(t) x-p, j(x-p)\right\rangle \leq & \|x-p\|^{2}-\lambda^{i}\left\|x-T_{i}^{n}(t) x\right\|^{2} \\
& +\mu_{i n} \phi_{i}(\|x-p\|)+\xi_{\text {in }}, \quad \forall n \geq 1,
\end{aligned}
$$

where $\phi_{i}:[0, \infty) \rightarrow[0, \infty)$ is a continuous and strictly increasing function with $\phi_{i}(0)=0$, and

$$
\left\|T_{i}^{n}(t) x-T_{i}^{n}(t) y\right\| \leq L^{i}\|x-y\|, \quad \forall n \geq 1 .
$$

Consider a family $\left\{\Im_{i}\right\}_{i=1}^{N}$ of uniformly $L_{i}(t)$-Lipschitzian and $\left(\lambda_{i}(t),\left\{\mu_{i n}\right\},\left\{\xi_{i n}\right\}, \phi_{i}\right)$-total asymptotically strict pseudocontractive semigroups of $C$ and let

$$
L:=\max _{1 \leq i \leq N} L^{i}<\infty, \quad \lambda:=\min _{1 \leq i \leq N} \lambda^{i}>0, \quad F:=\bigcap_{1 \leq i \leq N} \bigcap_{t \geq 0} F\left(T_{i}(t)\right) \neq \emptyset,
$$




$$
\mu_{n}=\max _{1 \leq i \leq N} \mu_{i n}, \quad \xi_{n}=\max _{1 \leq i \leq N} \xi_{i n}, \quad \phi=\max _{1 \leq i \leq N} \phi_{i} .
$$

For $x, y \in C, p \in F, t \geq 0$ and any $i \in\{1,2, \ldots, N\}$,

$$
\begin{aligned}
\left\langle T_{i}^{n}(t) x-p, j(x-p)\right\rangle \leq & \|x-p\|^{2}-\lambda\left\|x-T_{i}^{n}(t) x\right\|^{2} \\
& +\mu_{n} \phi(\|x-p\|)+\xi_{n}, \quad \forall n \geq 1
\end{aligned}
$$

and

$$
\left\|T_{i}^{n}(t) x-T_{i}^{n}(t) y\right\| \leq L\|x-y\|, \quad \forall n \geq 1 .
$$

Now, we are ready to give our main results.

Theorem 2.1 Let E be a real Banach space, and let $C$ be a nonempty, closed convex subset of E. Let $\left\{\Im_{i}\right\}_{i=1}^{N}$ be a finite family of uniformly $L_{i}(t)$-Lipschitzian and $\left(\lambda_{i}(t),\left\{\mu_{i n}\right\},\left\{\xi_{\text {in }}\right\}, \phi_{i}\right)$ total asymptotically strictly pseudocontractive semigroups of $C, L_{i}(t), \lambda_{i}(t),\left\{\mu_{i n}\right\},\left\{\xi_{i n}\right\}, \phi_{i}$, $L^{i}, \lambda^{i}, F^{i}, L, \lambda, F, \phi,\left\{\mu_{n}\right\}$ and $\left\{\xi_{n}\right\}$ be the same as above. In addition, there exist positive constants $M$ and $M^{*}$ such that $\phi(\lambda) \leq M^{*} \lambda^{2}$ for all $\lambda \geq M$. Let $\left\{x_{n}\right\}$ be the sequence generated by

$$
\begin{aligned}
& x_{1} \in C, \\
& x_{n+1}=\left(1-\alpha_{n}\right) x_{n}+\alpha_{n} T_{n}^{n}\left(t_{n}\right) x_{n}, \quad n \geq 1,
\end{aligned}
$$

where $T_{n}^{n}\left(t_{n}\right)=T_{n(\bmod N)}^{n}\left(t_{n}\right), \forall n \geq 1,\left\{\alpha_{n}\right\}$ is a sequence in $(0,1)$ and $\left\{t_{n}\right\}$ is an increasing sequence in $[0, \infty)$. If the following conditions are satisfied:

(1) $\sum_{n=1}^{\infty} \alpha_{n}^{2}<\infty, \sum_{n=1}^{\infty} \alpha_{n}=\infty, \sum_{n=1}^{\infty} \alpha_{n} \mu_{n}<\infty, \sum_{n=1}^{\infty} \alpha_{n} \xi_{n}<\infty$.

(2) Assume for any $i \in\{1,2, \ldots, N\}$ and for any bounded subset $D \subset C$,

$$
\lim _{n \rightarrow \infty} \sup _{x \in D, s \in R^{+}}\left\|T_{i}^{n}\left(s+t_{n}\right) x-T_{i}^{n}\left(t_{n}\right) x\right\|=0
$$

(3) There exists a compact subset $G$ of E such that $\bigcap_{t \geq 0} T_{l}(t)(C) \subset G$ for some

$$
l \in\{1,2, \ldots, N\} \text {. }
$$

Then the sequence $\left\{x_{n}\right\}$ converges strongly to some element in F.

Proof The proof of Theorem 2.1 is divided into four steps.

Step 1. First we prove that $\lim _{n \rightarrow \infty}\left\|x_{n}-p\right\|$ exists for all $p \in F$.

For any $p \in F$, by (1.4) we have

$$
\left\|T_{n}^{n}\left(t_{n}\right) x_{n}-p\right\|=\left\|T_{n}^{n}\left(t_{n}\right) x_{n}-T_{n}^{n}\left(t_{n}\right) p\right\| \leq L\left\|x_{n}-p\right\| .
$$

It follows from (2.7) and (2.9) that

$$
\begin{aligned}
\left\|x_{n+1}-p\right\| & =\left\|\left(1-\alpha_{n}\right) x_{n}+\alpha_{n} T_{n}^{n}\left(t_{n}\right) x_{n}-\left(1-\alpha_{n}\right) p-\alpha_{n} p\right\| \\
& \leq\left(1-\alpha_{n}\right)\left\|x_{n}-p\right\|+\alpha_{n}\left\|T_{n}^{n}\left(t_{n}\right) x_{n}-p\right\| \\
& \leq\left(1-\alpha_{n}\right)\left\|x_{n}-p\right\|+\alpha_{n} L\left\|x_{n}-p\right\| \\
& \leq(1+L)\left\|x_{n}-p\right\|
\end{aligned}
$$


and

$$
\begin{aligned}
\left\|x_{n+1}-x_{n}\right\| & =\alpha_{n}\left\|T_{n}^{n}\left(t_{n}\right) x_{n}-x_{n}\right\| \\
& \leq \alpha_{n}\left(\left\|T_{n}^{n}\left(t_{n}\right) x_{n}-p\right\|+\left\|x_{n}-p\right\|\right) \\
& \leq \alpha_{n}(1+L)\left\|x_{n}-p\right\| .
\end{aligned}
$$

By (1.10), for the point $x_{n+1}$ and $p$, there exists $j\left(x_{n+1}-p\right) \in J\left(x_{n+1}-p\right)$ such that

$$
\begin{aligned}
\left\langle T_{n}^{n}\left(t_{n}\right) x_{n+1}-x_{n+1}, j\left(x_{n+1}-p\right)\right\rangle \leq & -\lambda\left\|T_{n}^{n}\left(t_{n}\right) x_{n+1}-x_{n+1}\right\|^{2} \\
& +\mu_{n} \phi\left(\left\|x_{n+1}-p\right\|\right)+\xi_{n}, \quad \forall n \geq 1 .
\end{aligned}
$$

Since $\phi$ is an increasing function, it results that $\phi(\lambda) \leq \phi(M)$ if $\lambda \leq M$ and $\phi(\lambda) \leq M^{*} \lambda^{2}$ if $\lambda \geq M$. In either case, we can obtain that

$$
\phi(\lambda) \leq \phi(M)+M^{*} \lambda^{2}
$$

Thus, by Lemma 1.4, (2.10)-(2.13), we have

$$
\begin{aligned}
\left\|x_{n+1}-p\right\|^{2} \leq & \left\|x_{n}-p+\alpha_{n}\left(T_{n}^{n}\left(t_{n}\right) x_{n}-x_{n}\right)\right\|^{2} \\
\leq & \left\|x_{n}-p\right\|^{2}+2 \alpha_{n}\left\langle T_{n}^{n}\left(t_{n}\right) x_{n}-x_{n}, j\left(x_{n+1}-p\right)\right\rangle \\
= & \left\|x_{n}-p\right\|^{2}+2 \alpha_{n}\left\langle T_{n}^{n}\left(t_{n}\right) x_{n}-T_{n}^{n}\left(t_{n}\right) x_{n+1}, j\left(x_{n+1}-p\right)\right\rangle \\
& +2 \alpha_{n}\left\langle T_{n}^{n}\left(t_{n}\right) x_{n+1}-x_{n+1}, j\left(x_{n+1}-p\right)\right\rangle \\
& +2 \alpha_{n}\left\langle x_{n+1}-x_{n}, j\left(x_{n+1}-p\right)\right\rangle \\
\leq & \left\|x_{n}-p\right\|^{2}+2 \alpha_{n} L\left\|x_{n+1}-x_{n}\right\|\left\|x_{n+1}-p\right\| \\
& -2 \alpha_{n} \lambda\left\|T_{n}^{n}\left(t_{n}\right) x_{n+1}-x_{n+1}\right\|^{2}+2 \alpha_{n} \mu_{n} \phi\left(\left\|x_{n+1}-p\right\|\right) \\
& +2 \alpha_{n} \xi_{n}+2 \alpha_{n}\left\|x_{n+1}-x_{n}\right\|\left\|x_{n+1}-p\right\| \\
\leq & \left(1+2 \alpha_{n}^{2}(1+L)^{3}+2 \alpha_{n} \mu_{n} M^{*}(1+L)^{2}\right)\left\|x_{n}-p\right\|^{2} \\
& -2 \alpha_{n} \lambda\left\|T_{n}^{n}\left(t_{n}\right) x_{n+1}-x_{n+1}\right\|^{2} \\
& +2 \alpha_{n} \mu_{n} \phi(M)+2 \alpha_{n} \xi_{n} \\
\leq & \left(1+2 \alpha_{n}^{2}(1+L)^{3}+2 \alpha_{n} \mu_{n} M^{*}(1+L)^{2}\right)\left\|x_{n}-p\right\|^{2} \\
& +2 \alpha_{n} \mu_{n} \phi(M)+2 \alpha_{n} \xi_{n} .
\end{aligned}
$$

By condition (1), it follows from Lemma 1.3 that the limit $\lim _{n \rightarrow \infty}\left\|x_{n}-p\right\|$ exists and so the sequence $\left\{x_{n}\right\}$ is bounded in $C$.

Step 2. Now we prove that

$$
\liminf _{n \rightarrow \infty}\left\|x_{n}-T_{n}^{n}(t) x_{n}\right\|=0
$$


In fact, it follows from (2.14) that

$$
\begin{aligned}
2 \alpha_{n} \lambda\left\|T_{n}^{n}\left(t_{n}\right) x_{n+1}-x_{n+1}\right\|^{2} \leq & \left\|x_{n}-p\right\|^{2}-\left\|x_{n+1}-p\right\|^{2}+2 \alpha_{n}^{2}(1+L)^{3} K^{2} \\
& +\left(2 M^{\prime \prime}(1+L)^{2} K^{2}+2 \phi(M)\right) \alpha_{n} \mu_{n}+2 \alpha_{n} \xi_{n}
\end{aligned}
$$

where $K=\sup _{n \geq 1}\left\|x_{n}-p\right\|$. Hence, for some $m \geq 1$,

$$
\begin{aligned}
2 \lambda & \sum_{n=1}^{m} \alpha_{n}\left\|T_{n}^{n}\left(t_{n}\right) x_{n+1}-x_{n+1}\right\|^{2} \\
\leq & \sum_{n=1}^{m}\left(\left\|x_{n}-p\right\|^{2}-\left\|x_{n+1}-p\right\|^{2}\right)+2(1+L)^{3} K^{2} \sum_{n=1}^{m} \alpha_{n}^{2} \\
& +\left(2 M^{*}(1+L)^{2} K^{2}+2 \phi(M)\right) \sum_{n=1}^{m} \alpha_{n} \mu_{n}+2 \sum_{n=1}^{m} \alpha_{n} \xi_{n} \\
\leq & \left\|x_{1}-p\right\|^{2}+2(1+L)^{3} K^{2} \sum_{n=1}^{m} \alpha_{n}^{2} \\
& +\left(2 M^{*}(1+L)^{2} K^{2}+2 \phi(M)\right) \sum_{n=1}^{m} \alpha_{n} \mu_{n}+2 \sum_{n=1}^{m} \alpha_{n} \xi_{n} .
\end{aligned}
$$

Letting $m \rightarrow \infty$, we have

$$
\begin{aligned}
2 \lambda & \sum_{n=1}^{\infty} \alpha_{n}\left\|T_{n}^{n}\left(t_{n}\right) x_{n+1}-x_{n+1}\right\|^{2} \\
\leq & \left\|x_{1}-p\right\|^{2}+2(1+L)^{3} K^{2} \sum_{n=1}^{\infty} \alpha_{n}^{2} \\
& +\left(2 M^{*}(1+L)^{2} K^{2}+2 \phi(M)\right) \sum_{n=1}^{\infty} \alpha_{n} \mu_{n}+2 \sum_{n=1}^{\infty} \alpha_{n} \xi_{n} .
\end{aligned}
$$

By condition (1), we obtain

$$
\sum_{n=1}^{\infty} \alpha_{n}\left\|T_{n}^{n}\left(t_{n}\right) x_{n+1}-x_{n+1}\right\|^{2}<\infty,
$$

which implies

$$
\liminf _{n \rightarrow \infty}\left\|x_{n+1}-T_{n}^{n}\left(t_{n}\right) x_{n+1}\right\|=0 .
$$

Since $\lim _{n \rightarrow \infty}\left\|x_{n}-p\right\|$ exists for all $p \in F$ and $\lim _{n \rightarrow \infty} \alpha_{n}=0$, using (2.11), we have

$$
\lim _{n \rightarrow \infty}\left\|x_{n+1}-x_{n}\right\|=0
$$

This implies that

$$
\lim _{n \rightarrow \infty}\left\|x_{n+i}-x_{n}\right\|=0, \quad \forall i \in\{1,2, \ldots, N\}
$$


It follows from (2.20) and (2.21) that

$$
\begin{aligned}
& \liminf _{n \rightarrow \infty}\left\|x_{n}-T_{n}^{n}\left(t_{n}\right) x_{n}\right\| \\
& \quad \leq \liminf _{n \rightarrow \infty}\left\{\left\|x_{n}-x_{n+1}\right\|+\left\|x_{n+1}-T_{n}^{n}\left(t_{n}\right) x_{n+1}\right\|+\left\|T_{n}^{n}\left(t_{n}\right) x_{n+1}-T_{n}^{n}\left(t_{n}\right) x_{n}\right\|\right\} \\
& \quad \leq \liminf _{n \rightarrow \infty}\left\{(1+L)\left\|x_{n}-x_{n+1}\right\|+\left\|x_{n+1}-T_{n}^{n}\left(t_{n}\right) x_{n+1}\right\|\right\}=0 .
\end{aligned}
$$

For any $t \geq 0$, we have

$$
\begin{aligned}
& \left\|x_{n}-T_{n}^{n}(t) x_{n}\right\| \\
& \quad \leq\left\|x_{n}-T_{n}^{n}\left(t_{n}\right) x_{n}\right\|+\left\|T_{n}^{n}\left(t_{n}\right) x_{n}-T_{n}^{n}\left(t_{n}+t\right) x_{n}\right\|+\left\|T_{n}^{n}\left(t_{n}+t\right) x_{n}-T_{n}^{n}(t) x_{n}\right\| \\
& \quad \leq(1+L)\left\|x_{n}-T_{n}^{n}\left(t_{n}\right) x_{n}\right\|+\sup _{z \in\left\{x_{n}\right\}, t \in R^{+}}\left\|T_{n}^{n}\left(t+t_{n}\right) z-T_{n}^{n}\left(t_{n}\right) z\right\|,
\end{aligned}
$$

from (2.8) and (2.23), conclusion (2.15) is proved.

Step 3. Now we prove that

$$
\liminf _{n \rightarrow \infty}\left\|x_{n}-T_{m}(t) x_{n}\right\|=0, \quad \forall m \in\{1,2, \ldots, N\}
$$

For each $j=1,2, \ldots, N$, let

$$
\eta_{i N+j}:=\left\|x_{i N+j}-T_{j}^{i N+j}(t) x_{i N+j}\right\| .
$$

Since $T_{j}$ is uniformly $L_{j}(t)$-Lipschitzian continuous, it follows from (2.1) and (2.4) that

$$
\begin{aligned}
&\left\|x_{i N+j}-T_{j}(t) x_{i N+j}\right\| \\
& \leq\left\|x_{i N+j}-T_{j}^{i N+j}(t) x_{i N+j}\right\|+\left\|T_{j}^{i N+j}(t) x_{i N+j}-T_{j}(t) x_{i N+j}\right\| \\
& \leq \eta_{i N+j}+L\left\|T_{j}^{i N+j-1}(t) x_{i N+j}-x_{i N+j}\right\| \\
& \leq \eta_{i N+j}+L\left\{\left\|T_{j}^{i N+j-1}(t) x_{i N+j}-T_{j}^{i N+j-1}(t) x_{i N+j-1}\right\|\right. \\
&\left.\quad+\left\|T_{j}^{i N+j-1}(t) x_{i N+j-1}-x_{i N+j-1}\right\|+\left\|x_{i N+j-1}-x_{i N+j}\right\|\right\} \\
& \leq \eta_{i N+j}+L(1+L)\left\|x_{i N+j}-x_{i N+j-1}\right\|+L \eta_{i N+j-1},
\end{aligned}
$$

from (2.15) and (2.21), we have

$$
\liminf _{n \rightarrow \infty}\left\|x_{i N+j}-T_{j}(t) x_{i N+j}\right\|=0
$$

This implies that

$$
\liminf _{n \rightarrow \infty}\left\|x_{n}-T_{n}(t) x_{n}\right\|=0
$$

where $T_{n}(t)=T_{n(\bmod N)}(t), \forall n \geq 1$. 
For $l \in\{1,2, \ldots, N\}$, we have

$$
\begin{aligned}
& \left\|x_{n}-T_{n+l}(t) x_{n}\right\| \\
& \quad \leq\left\|x_{n}-x_{n+l}\right\|+\left\|x_{n+l}-T_{n+l}(t) x_{n+l}\right\|+\left\|T_{n+l}(t) x_{n+l}-T_{n+l}(t) x_{n}\right\| \\
& \quad \leq(1+L)\left\|x_{n}-x_{n+l}\right\|+\left\|x_{n+l}-T_{n+l}(t) x_{n+l}\right\|,
\end{aligned}
$$

from (2.22) and (2.27), we obtain

$$
\liminf _{n \rightarrow \infty}\left\|x_{n}-T_{n+l}(t) x_{n}\right\|=0
$$

It follows from (2.28) that $\liminf _{n \rightarrow \infty}\left\|x_{n}-T_{m}(t) x_{n}\right\|=0, \forall m \in\{1,2, \ldots, N\}$. This completes the proof.

Step 4. Finally, we prove the sequence $\left\{x_{n}\right\}$ converges strongly to some element in $F$.

By (2.25), we have $\liminf _{n \rightarrow \infty}\left\|x_{n}-T_{m}(t) x_{n}\right\|=0, \forall m \in\{1,2, \ldots, N\}$. If $\bigcap_{t \geq 0} T_{l}(t)(C) \subset G$ for some compact subset $G$ of $E$ and some $l \in\{1,2, \ldots, N\}$, then there exists a subsequence $\left\{x_{n_{k}}\right\}$ of $\left\{x_{n}\right\}$ and $q \in C$ such that

$$
\lim _{k \rightarrow \infty} T_{l}(t) x_{n_{k}}=q, \quad \lim _{k \rightarrow \infty}\left\|x_{n_{k}}-T_{l}(t) x_{n_{k}}\right\|=0
$$

Hence, it follows from (2.29) that

$$
\lim _{k \rightarrow \infty} x_{n_{k}}=q
$$

Now, for any $m \in\{1,2, \ldots, N\}$, since $\liminf _{k \rightarrow \infty}\left\|x_{n_{k}}-T_{m}(t) x_{n_{k}}\right\|=0$, there exists a subsequence $\left\{x_{n_{k_{j}}}\right\}$ of $\left\{x_{n_{k}}\right\}$ such that $\lim _{j \rightarrow \infty}\left\|x_{n_{k_{j}}}-T_{m}(t) x_{n_{k_{j}}}\right\|=0$. Using (2.30) and the fact that $T_{m}$ is Lipschitzian, we get $q \in \bigcap_{t \geq 0} F\left(T_{m}(t)\right)$. Since $m \in\{1,2, \ldots, N\}$ is arbitrarily chosen, we have $q \in F$.

Since $x_{n_{k}} \rightarrow q$ as $k \rightarrow \infty$ and the limit $\lim _{n \rightarrow \infty}\left\|x_{n}-q\right\|$ exists, this implies that $x_{n} \rightarrow q \in$ $F$ as $n \rightarrow \infty$. This completes the proof.

The following theorem can be obtained from Theorem 2.1 immediately.

Theorem 2.2 Let E be a real Banach space, and let $C$ be a nonempty, closed convex subset of E. Let $\left\{\mathfrak{\Im}_{i}\right\}_{i=1}^{N}$ be a finite family of uniformly $L_{i}(t)$-Lipschitzian and $\left(\left\{k_{i n}\right\}, \lambda_{i}(t)\right)$-asymptotically strictly pseudocontractive semigroups of $C, L_{i}(t), \lambda_{i}(t), L^{i}, \lambda^{i}, F^{i}, L, \lambda, F$ be as in Theorem 2.1. $k_{n}=\max _{1 \leq i \leq N}\left\{k_{i n}\right\}$. Let $\left\{x_{n}\right\}$ be the sequence defined by (2.7), $\left\{\alpha_{n}\right\}$ is a sequence in $(0,1)$ and let $\left\{t_{n}\right\}$ be an increasing sequence in $[0, \infty)$. If the following conditions are satisfied:

(1) $\sum_{n=1}^{\infty} \alpha_{n}=\infty, \sum_{n=1}^{\infty} \alpha_{n}^{2}<\infty, \sum_{n=1}^{\infty} \alpha_{n}\left(k_{n}-1\right)<\infty$.

(2) Assume for any $i \in\{1,2, \ldots, N\}$ and for any bounded subset $D \subset C$,

$$
\lim _{n \rightarrow \infty} \sup _{x \in D, s \in R^{+}}\left\|T_{i}^{n}\left(s+t_{n}\right) x-T_{i}^{n}\left(t_{n}\right) x\right\|=0
$$

(3) There exists a compact subset $G$ of E such that $\bigcap_{t \geq 0} T_{l}(t)(C) \subset G$ for some $l \in\{1,2, \ldots, N\}$. 
Then the sequence $\left\{x_{n}\right\}$ converges strongly to some element in $F$.

Proof Taking $\phi(\lambda)=\lambda^{2}, \xi_{n}=0, \mu_{n}=k_{n}-1$ in Theorem 2.1, since all conditions in Theorem 2.1 are satisfied. It follows from Theorem 2.1 that the sequence $x_{n} \rightarrow q \in F:=$ $\bigcap_{t \geq 0} F(T(t))$ as $n \rightarrow \infty$.

This completes the proof of Theorem 2.2.

Remark 2.3 Theorems 2.1 and 2.2 extend and improve the corresponding results of Chang et al. [11], Shioji and Takahashi [1], Suzuki [2], Xu [3], Aleyner and Reich [4] and others.

Open problem It may be interesting to post the following open problem: Can Theorem 2.1 be generalized to a finite family of semigroups of mappings $S$ which are representation, so commutative or left reversible (see Liu and Zhang [13] and related references there), which is not necessarily the positive real number with addition?

Competing interests

The authors declare that they have no competing interests.

Authors' contributions

All authors read and approved the final manuscript.

\section{Author details}

${ }^{1}$ School of Science, South West University of Science and Technology, Mianyang, Sichuan 621002, China. ${ }^{2}$ College of Statistics and Mathematics, Yunnan University of Finance and Economics, Kunming, Yunnan 650221, China.

\section{Acknowledgements}

The authors would like to express their thanks to the editor and the referees for their helpful comments and suggestions. This work was supported by the Natural Science Foundation of Sichuan Province (No. 08ZA008).

Received: 9 October 2012 Accepted: 19 June 2013 Published: 8 July 2013

\section{References}

1. Shioji, N, Takahashi, W: Strong convergence theorems for asymptotically nonexpansive mappings in Hilbert spaces. Nonlinear Anal. 34(1), 87-99 (1998)

2. Suzuki, T: On strong convergence to a common fixed point of nonexpansive semigroups in Hilbert spaces. Proc. Am. Math. Soc. 131(7), 2133-2136 (2003)

3. Xu, HK: Strong convergence theorem for contraction semigroups in Banach spaces. Bull. Aust. Math. Soc. 72(3), 371-379 (2005)

4. Aleyner, A, Reich, S: An explicit construction of sunny nonexpansive retractions in Banach spaces. Fixed Point Theory Appl. 3, 295-305 (2005)

5. Zhang, SS, Yang, L, Liu, JA: Strong convergence theorem for nonexpansive semigroups in Banach spaces. Appl. Math. Mech. 28(10), 1287-1297 (2007)

6. Li, S, Li, LH, Su, F: General iteration methods for a one-parameter nonexpansive semigroups in Hilbert spaces. Nonlinear Anal. 70(9), 3065-3071 (2009)

7. Zhang, SS, Yang, L, Lee, HWJ, Chan, CK: Strong convergence theorem for nonexpansive semigroups in Hilbert spaces. Acta Math. Sin. 52(2), 337-342 (2009)

8. Suzuki, T: Fixed point property for nonexpansive mappings versus that for nonexpansive semigroups. Nonlinear Anal. 70, 3358-3361 (2009)

9. Zhang, SS: Convergence theorem of common fixed points for Lipschitzian pseudocontraction semigroups in Banach spaces. Appl. Math. Mech. 30, 145-152 (2009)

10. Chang, SS, Chan, CK, Lee, HWJ, Yang, L: A system of mixed equilibrium problems, fixed point problems of strictly pseudocontractive mappings and nonexpansive semigroups. Appl. Math. Comput. 216(1), 51-60 (2010)

11. Chang, SS, Cho, YJ, Lee, HWJ, Chan, CK: Strong convergence theorems for Lipschitzian demicontraction semigroups in Banach spaces. Fixed Point Theory Appl. (2011). doi:10.1155/2011/583423

12. Chang, SS: On Chidume's open questions and approximate solutions for multi-valued strongly accretive mapping equations in Banach spaces. J. Math. Anal. Appl. 216, 94-111 (1997)

13. Lau, AT-M, Zhang, Y: Fixed point properties of semigroups of non-expansive mappings. J. Funct. Anal. 254(10), 2534-2554 (2008) 